\title{
Parametric Analysis of Ring Gear Structure Vibration Modes
}

\author{
Romil P. Tanna \\ Department of Mechanical Engineering, The University of Alabama, \\ 290 Hardaway Hall, Box 870276, Tuscaloosa, AL 35487, USA
}

Teik C. Lim $^{\dagger}$

University of Cincinnati, Department of Mechanical, Industrial and Nuclear Engineering,

598 Rhodes Hall, P. O. Box 210072, Cincinnati, OH 45221, USA

(Received 6 October 2002; accepted 9 January 2006)

\begin{abstract}
A comprehensive parametric analysis to quantify the sensitivity of planetary ring gear structural modes to variations in its geometrical parameters is performed by applying the dynamic finite element method. Specifically, the effects of the rim thickness to radius ratio, rim thickness to width ratio, helix angle, number of teeth, number of splines, and spline height and angular width are examined. The eigen-solutions reveal four classes of commonly occurring radial inextensional, extensional, out-of-plane bending and torsional ring modes. Of these four groups of modes, the radial inextensional and out-of-plane bending, frequencies are generally lower and fall more within the undesirable gear whine spectrum caused by the transmission error excitation. The predicted natural frequency sensitivities to the ring gear design parameters of interest are compiled graphically. The resulting trends are useful in identifying more robust designs with a lesser tendency towards the generation and transmissibility of excessive gear noise levels.
\end{abstract}

${ }^{\dagger}$ Member of the International Institute of Acoustics and Vibration (IIAV)

\section{INTRODUCTION}

Planetary gear trains (PGT) are widely applied in drivetrain systems of automotive, aerospace, marine, and machine tool industries. This is because of their numerous advantages over simple countershaft gear drives, including larger speed reduction, greater torque/weight ratios, lower bearing load densities, compactness, etc. However, due to their high speed and load applications, PGT can be quite susceptible to undesirable noise and vibration caused by the rotating carrier/planet gear dynamic mesh forces. These forces and the resultant vibrations often lead to gear whine and other durability problems degrading the performances of the drivetrain. Since one of the primary structure-borne vibratory paths is through the ring gear structure, the flexibility of the ring gear and its resonance modes could amplify the transmitted dynamic response. Hence, dynamic analysis including the predictions of natural modes and dynamic gear mesh forces have been a major focus in the study of PGT as discussed below.

Most of the published research work on PGT dynamics assumes a rigid ring gear model with localised mesh stiffness, and the literature contains an abundance of analysis carried out based on this concept. ${ }^{1-6}$ In addition, other factors such as dynamic transmission error, manufacturing uncertainties, frictional forces, torque variations, and mesh stiffness have been studied too. ${ }^{1-3}$ For example, an experimental study completed by Hidaka et al. examined the effect of torque variation due to mesh and run-out errors. ${ }^{1}$ Kahraman developed a nonlinear time-varying dynamic model of a planetary transmission with an arbitrary number of planets. ${ }^{2}$ The model includes manufacturing variations, pinion spacing arrangements, tooth separation, and time-varying gear mesh stiffness. Kahraman and Blankenship investigated the effect of planet tooth mesh phasing on the static and dynamic characteristics of epicyclic gear sets. ${ }^{3}$ Kahraman also formulated a torsional model to predict the natural frequencies of a PGT set for use in the early design stage when only a few parameters are known. ${ }^{4}$ Saada and Velex studied the influence of mesh stiffness, helix angle and ring support stiffness on planetary gear response. ${ }^{5}$ Velex and Flamand extended the theory to examine the effect of mesh parametric excitation. ${ }^{6}$ In 1999 , Lin and Parker investigated the natural frequency and vibration mode sensitivities of PGTs to system parameters like support and mesh stiffness, component masses, and mass moment of inertias. ${ }^{7}$ A year later, they used the same model to determine the effect of unequally spaced planets, ${ }^{8}$ and the effectiveness of planet phasing for vibration suppression. ${ }^{9}$ Many of these models appear to predict dynamic mesh forces and overall system modes quite accurately. However, the fundamental assumption of rigid ring gear renders these models incapable of predicting the extent of force and vibration transmissibility through the ring gear structure, and determining the effects of ring gear modes. In the same year, Parker et al. used a finite element (FE) model that incorporates contact mechanics to examine the dynamic response of a helicopter planetary gear system over a wide range of operating speeds and torques. ${ }^{10}$ Even though a 2-dimensional FE model was developed to include gear flexibilities, the outer boundary of the ring gear was rigidly constrained. This prevented the model from predicting the vibration transmissibility through the ring gear. In fact, a thorough literature survey has revealed very few articles that consider the effect of ring gear flexibility. In one study, Ma and Botman analysed the 